CAMILA Pigatto 1

Caroline Mombaque dos Santos ${ }^{1}$

Wendel Mombaque dos Santos ${ }^{2}$

Walter Santos Neme 3

Luiz Osório Cruz Portela ${ }^{4}$

EDSON NUNES DE MORAES ${ }^{5}$

Francisco MaXimILIANO PANCICH GaLLARRETA ${ }^{5}$

\title{
Efeito do exercício físico sobre os
}

parâmetros hemodinâmicos

\section{Effects of physical exercise on the fetal hemodynamic parameters}

Cartas ao editor representam a correspondência entre diversos autores e os leitores, por meio dos editores das revistas, oferecendo não apenas a oportunidade de debater em um fórum aberto, mas também de contribuir para a validação da pesquisa ${ }^{1}$. Assim, agradecemos a oportunidade neste espaço da revista.

O objetivo do estudo realizado pelo nosso grupo de pesquisa foi testar o efeito do exercício físico agudo na hemodinâmica feto-placentária (artérias uterinas, umbilicais e cerebral média e ducto venoso) de pacientes com gestação saudável, a fim de instigar os profissionais da saúde sobre o tema. Tal pesquisa visou testar pontualmente esses efeitos e, como redigimos em nosso artigo, objetivou-se também encorajar outros grupos de pesquisa para avaliar se o exercício físico contínuo em gestantes previamente sedentárias não trará repercussões fetais.

Os pesquisadores envolvidos nessa pesquisa entendem que o Grupo Controle das pacientes submetidas ao exercício físico é feito pela própria paciente na análise prévia ao mesmo, sendo que cada uma, como descrito em todo texto, foi avaliada pré- e pós-protocolo de pesquisa. Como dispomos largamente na literatura de dados dopplervelocimétricos durante a gestação de todos os seguimentos vasculares analisados nessa pesquisa e todos foram realizados em repouso, não nos parece claro o porquê de estudar um Grupo Controle que não realize exercício se tais resultados já estão estabelecidos² ${ }^{2}$.

A leitura atenta do manuscrito indica ao leitor que dez gestantes participaram do estudo, sendo que cada uma foi avaliada durante dois períodos gestacionais distintos, totalizando 20 observações no presente estudo.

O fato de selecionarmos uma amostra de conveniência significa que os elementos foram escolhidos por um julgamento de valor, e não por questões de aleatoriedade estatística. Este tipo de seleção resume a experiência local, servindo como uma aproximação para o que acontece em instituições com características semelhantes e úteis para verificar se um problema existe no universo em que estão inseridas, além de que os resultados obtidos a partir desse tipo de amostragem servirão de base para o trabalho de outros pesquisadores ${ }^{3}$.

A mensuração biométrica fetal foi efetuada pela equipe de pesquisadores em todas as participantes do estudo. Portanto, entre os critérios de exclusão da pesquisa estão alterações estruturais, patologias maternas que predispõem a alterações como macrossomia e restrição de crescimento fetal, sendo incluídas no estudo apenas gestantes que se

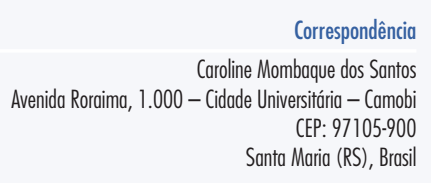

DOI: $10.1590 / 50100-720320140005229$
IServiço de Medicina Fetal da Universidade Federal de Santa Maria - UFSM - Santa Maria (RS), Brasil.

2Programa de Pós-graduação em Enfermagem da Universidade Federal de Santa Maria - UFSM - Santa Maria (RS), Brasil. ${ }^{3}$ Hospital Universitário de Santa Maria da Universidade Federal de Santa Maria - UFSM - Santa Maria (RS), Brasil. ${ }^{4}$ Centro de Educação Física e Desportos da Universidade Federal de Santa Maria - UFSM - Santa Maria (RS), Brasil. ${ }^{5}$ Departamento de Ginecologia e Obstetrícia da Universidade Federal de Santa Maria - UFSM - Santa Maria (RS), Brasil. 
enquadrassem na avaliação de gestação sem complicações, logo de baixo risco. Esse fato determinante da população estudada responde ao questionamento de que fetos com alterações no padrão de crescimento poderiam ser fatores confundidores dos resultados, porém, nesse estudo, tais fetos não fizeram parte do grupo de estudo, sendo o viés referido inverídico.

O tempo médio de fadiga voluntária atingido pelas pacientes do estudo foi de 12 minutos na primeira intervenção e de 11 na segunda, notando-se semelhança mesmo em idade gestacional mais avançada, na qual o volume abdominal poderia atrapalhar a atividade. Com relação à Escala de Borg, esta foi utilizada pelo grupo de pesquisa e faz parte dos resultados de um outro manuscrito publicado pelos pesquisadores ${ }^{4}$, que pela grandeza de variáveis geradas pelo estudo estão separadas conforme objetivos específicos de pesquisa, sendo comum entres grupos de pesquisas a distribuição de dados em artigos diversos inclusive pela restrição de páginas, número de palavras e tabelas para a submissão de artigos científicos.

Concordamos com relação ao fato de que a hemodinâmica é mutável momentaneamente. Contudo, a dificuldade técnica da obtenção ao ultrassom de ondas de velocidade de fluxo durante a atividade proposta pelo protocolo de pesquisa seria óbvia e mais prejudicial do que a escolhida como método pelo grupo (a de avaliarmos pós-exercício em período inferior a cinco minutos), além de que outros estudos estabelecem o mesmo método ${ }^{5,6}$.

A referência técnica utilizada para a obtenção adequada dos dados de dopplervelocimetria dos seguimentos vasculares do estudo foi a proposta pelo "ISUOG - Practice Guidelines: use of Doppler ultrasonography in obstetrics", nesse mesmo protocolo encontra-se a recomendação e os motivos para que seja efetuada a avaliação da artéria umbilical no segmento de alça livre de cordão ${ }^{7}$.

A apresentação dos testes realizados na pesquisa, ou seja, paramétricos, demonstra por si só que a população do estudo obteve distribuição normal. Evita-se a inserção desses dados no manuscrito, pois não contribuem de forma significativa para a observação dos resultados obtidos. Sabe-se também que existem testes para populações com amostras pequenas, tal como o teste de Shapiro-Wilk, que foi utilizado no estudo referido ${ }^{8}$. Seguindo a linha de raciocínio, quando se trabalha com dados numéricos, observa-se uma tendência de se agruparem em torno de um valor central, sendo a média aritmética a melhor dessas medidas para dados simétricos e para quando não há valores aberrantes (muito extremos), como os dos resultados do estudo. Assim, a proposta do uso da mediana seria interessante se tivéssemos distribuições fortemente assimétricas dos dados e o intervalo interquartil também é uma medida descritiva de posição, mas não de dispersão conforme citado?.

A descrição de um conjunto de dados é mais completa quando se considera além de uma medida de tendência central, uma de dispersão, sendo o desvio padrão a utilizada para complementar os dados desse estudo já que permite uma interpretação direta da variação do grupo, por ser expresso na mesma unidade das medidas observadas ${ }^{9}$.

Buscando sempre melhorar a qualidade metodológica dos estudos por nós conduzidos, agradecemos os comentários e as sugestões. $\mathrm{O}$ fato de a classificação dos dados como não sendo clinicamente relevantes por não demostrarem alterações faz com que o grupo atinja o objetivo principal, ou seja, demonstrar que, em gestantes hígidas, o exercício físico pode - e deve - ser estimulado.

\section{Referências}

1. Amorim MM, Souza AS. A cultura da Carta ao Editor. Femina. 2013;41(1):1-4.

2. Sakamoto K. Construção de curvas de normalidade dos índices dopplervelocimétricos das circulações uteroplacentária, fetoplacentária e fetal [Tese]. São Paulo: Faculdade de Medicina; 2007 [citado 2014 set 07]. Disponível em: http://www.teses.usp.br/teses/ disponiveis/5/5139/tde-01062007-122759/

3. Pereira MG. Epidemiologia: teoria e prática. Rio de Janeiro: Guanabara Koogan; 1995.

4. dos Santos CM, dos Santos WM, Coutinho RX, Pigatto C, Portela LOC, Gallarreta FMP. A utilização da escala de Borg em atividade física com gestantes. ConScientiae Saúde. 2014;13241-5. [citado 2014 Set 07]. Disponível em: http://www.redalyc.org/articulo. oa? id $=92931451011$

5. de Oliveira Melo AS, Silva JL, Tavares JS, Barros VO, Leite DF, Amorim MM. Effect of a physical exercise program during pregnancy on uteroplacental and fetal blood flow and fetal growth: a randomized controlled trial. Obstet Gynecol. 2012;120(2 Pt 1):302-10.

6. Medeiros AA. [Influence of aerobic physical activity on blood pressure and fetal-placental blood flow in hypertensive pregnant women]. Rev Bras Ginecol Obstet. 2009;31 (4):211. Portuguese.

7. Bhide A, Acharya G, Bilardo CM, Brezinka C, Cafici D, HernandezAndrade E, et al. ISUOG Practice Guidelines: use of Doppler ultrasonography in obstetrics. Ultrasound Obstet Gynecol. 2013;41:233-9.

8. Razali NM, Wah YB. Power comparisons of Shapiro-wilk, Kolmogorov-smirnov, Lilliefors and Anderson-darling tests. J Stat Model Analytics. $2011 ; 2(1): 21-33$.

9. Moraes AB, Jacobi LF, Zanini RR. Estatística. N. 2. Série Naturais \& Exatas, Santa Maria: UFSM - CCNE; 2011. 